\title{
Health Status, Persistent Symptoms, and Effort Intolerance One Year After Acute COVID-19 Infection
}

\author{
Justin R. Kingery, MD,PhD ${ }^{1,2}$ (D), Monika M. Safford, $M D^{7}$, Paul Martin, $M D^{7}$, \\ Jennifer D. Lau, MPH' , Mangala Rajan, MBA', Graham T. Wehmeyer, $\mathrm{MD}^{3}$, \\ Han A. Li, MD ${ }^{3}$, Mark N. Alshak, MD ${ }^{3}$, Assem Jabri, MD ${ }^{7}$, Alina Kofman, MPA', \\ Christopher S. Babu, BS $/ B A^{3}$, Elizabeth K. Benitez, $B S^{3}$, Federico Palacardo, $B S^{3}$, \\ Indrani Guzman Das, $B A^{3}$, Kiara Kaylor, $B S^{3}$, Kwang M. Woo, $B A^{3}$, \\ Nicholas L. Roberts, $M P H^{2,3}$, Saher Rahiel, $B A^{7}$, Varshini Gali, $B A^{3}$, Lynn Han, BS ${ }^{3}$, \\ Joyce Lee, $B A^{3}$, Natalia Roszkowska, BA ${ }^{3}$, Yeo Eun Kim, BA ${ }^{3}$, Sufia Bakshi, BS ${ }^{3}$, \\ Cameron Hogan, BS ${ }^{4}$, Margaret McNairy, MD/MSc ${ }^{7,2}$, Laura C. Pinheiro, $P h D / M P H^{7}$, and \\ Parag Goyal, $\mathrm{MD}^{1,5}$
}

\begin{abstract}
'Division of General Internal Medicine, Department of Medicine, Weill Medical College of Cornell University, 402 E. 67th Street, 2 nd Floor, New York, NY, USA; ${ }^{2}$ Center for Global Health, Department of Medicine, Weill Medical College of Cornell University, New York, NY, USA; ${ }^{3}$ Weill Medical College of Cornell University, New York, NY, USA; ${ }^{4}$ Department of Statistics, Cornell University, Ithaca, NY, USA; ${ }^{5}$ Division of Cardiology, Department of Medicine, Weill Medical College of Cornell University, New York, NY, USA.
\end{abstract}

BACKGROUND: The long-term prevalence and risk factors for post-acute COVID-19 sequelae (PASC) are not well described and may have important implications for unvaccinated populations and policy makers.

OBJECTIVE: To assess health status, persistent symptoms, and effort tolerance approximately 1 year after COVID-19 infection

DESIGN: Retrospective observational cohort study using surveys and clinical data

PARTICIPANTS: Survey respondents who were survivors of acute COVID-19 infection requiring Emergency Department presentation or hospitalization between March 3 and May 15, 2020.

MAIN MEASURE(S): Self-reported health status, persistent symptoms, and effort tolerance

KEY RESULTS: The 530 respondents (median time between hospital presentation and survey 332 days [IQR 325-344]) had mean age $59.2 \pm 16.3$ years, $44.5 \%$ were female and $70.8 \%$ were non-White. Of these, $41.5 \%$ reported worse health compared to a year prior, $44.2 \%$ reported persistent symptoms, 36.2\% reported limitations in lifting/carrying groceries, 35.5\% reported limitations climbing one flight of stairs, 38.1\% reported limitations bending/kneeling/stooping, and $22.1 \%$ reported limitations walking one block. Even those without high-risk comorbid conditions and those seen only in the Emergency Department (but not hospitalized) experienced significant deterioration in health, persistent symptoms, and limitations in effort tolerance. Women (adjusted relative risk ratio [aRRR] 1.26, 95\% CI 1.01-1.56), those requiring mechanical ventilation (aRRR 1.48, 1.02-2.14), and people with HIV (aRRR 1.75, 1.14-2.69) were significantly more likely to report persistent symptoms. Age and other risk factors

Received October 21, 2021

Accepted December 17, 2021

Published online January 24, 2022 for more severe COVID-19 illness were not associated with increased risk of PASC.

CONCLUSIONS: PASC may be extraordinarily common 1 year after COVID-19, and these symptoms are sufficiently severe to impact the daily exercise tolerance of patients. PASC symptoms are broadly distributed, are not limited to one specific patient group, and appear to be unrelated to age. These data have implications for vaccine hesitant individuals, policy makers, and physicians managing the emerging longer-term yet unknown impact of the COVID-19 pandemic.

KEY WORDS: COVID-19; PASC; persistent symptoms.

J Gen Intern Med 37(5):1218-25

DOI: $10.1007 / \mathrm{s} 11606-021-07379-\mathrm{Z}$

(C) The Author(s) under exclusive licence to Society of General Internal Medicine 2022

\section{INTRODUCTION}

As we move further beyond the initial COVID-19 pandemic surge in the USA, there is increased interest in understanding the post-acute sequelae of COVID-19 (PASC). While advances in medical treatment such as glucocorticoid therapy have dramatically improved the morbidity and mortality in the acute disease phase, ${ }^{1}$ the long-term morbidity of COVID in survivors remains unknown. The few available studies indicate that symptoms may persist in the majority, with three-fourth of survivors reporting PASC symptoms at 60 days post-diagnosis ${ }^{2}$ and over one-half at 7-9 months. ${ }^{3,4}$ Risk factors for developing PASC are not well described. For example, whether baseline risk factors for severe COVID-19 are risk factors for PASC is unknown; it is also unknown whether risk for PASC differs according to the severity of COVID-19 infection. Answers to these questions are important especially for those members of the public considering 
whether to undergo vaccination to prevent COVID-19, since many younger, healthy individuals view their susceptibility to severe COVID-19 to be low.

In this study, we leveraged a well-characterized COVID-19 registry of patients in New York City ${ }^{5}$ to assess health status, persistent symptoms, and effort tolerance approximately 1 year after COVID-19 infection. We examined patterns based on baseline risk factors for severe COVID-19 infection, and on the severity of the acute COVID-19 infection. ${ }^{6}$ We also conducted a multivariable analysis to determine independent risk factors for PASC approximately 1 year after the acute COVID-19 episode.

\section{METHODS}

\section{Study Oversight}

This study was approved by the Weill Cornell Medicine Institutional Review Board, which waived informed consent.

\section{Study Setting}

New York-Presbyterian (NYP)/Weill Cornell Medical Center (WCMC) is a quaternary referral center and 862-bed teaching hospital located in the Upper East Side of Manhattan, NY; and NYP/Lower Manhattan Hospital (LMH) is an affiliated 180bed community hospital located in the Lower East Side of Manhattan, NY.

\section{Data Sources}

We conducted phone surveys of COVID-19 survivors derived from our COVID-19 registry which included all adults aged at least 18 years old who were hospitalized or presented to the Emergency Department (ED) with confirmed acute COVID19 within the NYP/WCMC-LMH hospital system in New York City between March 3 and May 15, 2020. All cases of COVID-19 were confirmed through real-time reverse-transcriptase polymerase chain reaction assays on nasopharyngeal swabs. Details of this registry have previously been described. ${ }^{5}$ Briefly, clinical data including baseline comorbid conditions were abstracted from medical records at the time of the index hospitalization for COVID-19. Patients who died or were discharged to hospice were excluded.

Twenty trained study team members contacted patients directly by phone at least 9 months after the discharge date. Specifically, we called patients up to a total of six times over 3 weeks. During the surveys, we asked respondents if their health was excellent, very good, good, fair, or poor, and if this was much better, somewhat better, about the same, somewhat worse, or much worse than one year ago (see Supplemental Figure 1 for the survey). Respondents were asked which was their most bothersome symptom (from a list including brain fog, cough, shortness of breath, sore throat, numbness/weakness, trouble concentrating, headache, muscle aches, nausea/ vomiting, diarrhea, sputum production, chest pain, abdominal pain, loss of smell/taste, insomnia), and also if they had symptoms in the past week (including loss of smell/taste, numbness or weakness, brain fog, chest pain, cough, shortness of breath, trouble concentrating, struggling to get to sleep or stay asleep). We used nine items assessing physical functioning from the Short Form 36-item survey.

\section{Statistical Analysis}

We compared characteristics of patients reporting symptoms to patients reporting no symptoms. We also compared survey responders and non-responders. Among responders, we tabulated PASC symptoms overall and by pre-COVID comorbid conditions (cardiopulmonary vs. non-cardiopulmonary vs. no conditions), and separately, by severity of acute COVID-19 (required mechanical ventilation, hospitalized but not requiring mechanical ventilation, or discharged directly from the Emergency Department). Persistent symptoms were defined as a patient report of at least one persistent symptom within the past 1 week. Patients with both cardiopulmonary and noncardiopulmonary comorbidities were classified as cardiopulmonary. To categorize comorbid conditions, we followed the Centers for Disease Control and Prevention classification of chronic conditions reported to increase risk of severe COVID$19 .{ }^{7}$ High-risk cardiopulmonary conditions included hypertension, coronary artery disease (CAD), heart failure, chronic obstructive pulmonary disorder (COPD) or interstitial lung disease (ILD), asthma, and current tobacco use. High-risk non-cardiopulmonary conditions included active cancer, renal disease, HIV, diabetes, obesity, pregnancy, transplant, history of stroke, and liver disease.

We calculated means and standard deviations (SD) for continuous variables and counts and percentages for categorical variables. Wilcoxon rank-sum tests were used to compare continuous variables across groups. For categorical variables, we used chi-square and Fisher's exact tests to evaluate differences across groups.

We conducted modified Poisson regression analysis with robust standard errors to identify independent predictors of any persistent symptoms. ${ }^{8}$ Candidate variables for inclusion in the models were obtained at the time of the index hospital visit and included demographic characteristics (age at the time of COVID-19 diagnosis, sex, race/ethnicity, neighborhood poverty rate defined by the percent of families in poverty within a patient's zip code), baseline comorbid conditions (asthma, COPD or ILD, heart failure, coronary artery disease, hypertension, active smoking, active cancer, renal disease, diabetes, HIV, obesity (body mass index $\geq 30 \mathrm{~kg} / \mathrm{m}^{2}$ ), pregnancy, any prior organ transplant, liver disease, and prior stroke), and severity of acute COVID-19 illness (ED only presentation vs. hospitalization without mechanical ventilation vs. hospitalization with mechanical ventilation). Statistical models were built using complete case analysis, and 79 cases with missing data were excluded. Relative risks and $95 \%$ confidence intervals $(95 \% \mathrm{CI})$ were calculated for each estimate. All analyses 
were conducted in R version 3.62 and SAS software version 9.4 (SAS Institute, Cary, NC) with 2-sided statistical tests and significance levels of 0.05 .

\section{RESULTS}

\section{Sample Characteristics}

Of the 1,032 eligible patients, we successfully contacted 633 (61.3\%), of which $103(10.0 \%)$ declined to participate. Therefore, 530 patients were analyzed. Median time between the presentation with COVID-19 and survey administration was 332 days (IQR 325-344). Table 1 shows baseline characteristics of patients who reported PASC symptoms at the time of the survey contrasted with those who did not. PASC patients were of similar age, sex, and race as non-PASC patients, but were significantly more likely to have had obesity and/or require hospitalization with mechanical ventilation and less likely to have had either renal or cerebrovascular disease prior to COVID-19 diagnosis. Notably, PASC patients had a similar prevalence of current smoking, poverty, and all other baseline co-morbidities as compared to those without PASC.

Compared to survey completers, the 502 non-completers were more frequently Black, more frequently active smokers, and more likely to have had cancer at the time of COVID-19 diagnosis as compared to those who did not complete the survey - other variables including age, sex, and poverty rate were similar between groups (Supplemental Table 1).

\section{Health Status}

Fully $41.5 \%$ of survey completers reported worse health compared to a year ago $(27.5 \%$ somewhat worse and $14.0 \%$ much worse). The proportion that reported worse health was similar across baseline comorbidity strata (Figure 1A) and severity of acute COVID-19 illness strata (Figure 1B).

\section{Persistent Symptoms}

Almost half of the cohort (44.2\%) reported persistent symptoms consistent with PASC. Persistent symptoms were similar across baseline comorbidity strata (Figure 1A). As compared to patients discharged from the ED, those hospitalized but not requiring mechanical ventilation reported a similar prevalence of PASC; however, hospitalized patients requiring mechanical ventilation were more likely to report PASC symptoms as compared to those discharged from the ED (RR 1.47 [CI 1.10-1.98]) (Figure 1B). Notably, the group with the lowest prevalence of PASC symptoms was patients with noncardiopulmonary conditions at baseline. The prevalence of the "most bothersome" symptoms reported were shortness of breath $(10.4 \%)$, fatigue $(5.5 \%)$, numbness in hands or feet (neuropathy) $(5.1 \%)$ and brain fog (3.8\%). Table 2 shows

Table 1 Baseline Characteristics of Participants Stratified by Presence of PASC Symptoms

\begin{tabular}{|c|c|c|c|c|}
\hline & $\begin{array}{l}\text { All } \\
(n=530)\end{array}$ & $\begin{array}{l}\text { No PASC } \\
(n=296)\end{array}$ & $\begin{array}{l}\text { PASC } \\
(n=234)\end{array}$ & $p$-value \\
\hline Mean age $(\mathrm{SD})$ - years & $59.2(16.2)$ & $59.9(16.6)$ & $58.2(15.7)$ & 0.21 \\
\hline Female-no. $(\%)$ & $236(44.5 \%)$ & $124(41.9 \%)$ & $112(47.9 \%)$ & 0.20 \\
\hline Race/ethnicity-no. (\%) & & & & 0.11 \\
\hline White & $155(29.2 \%)$ & $82(27.7 \%)$ & $73(31.2 \%)$ & \\
\hline Hispanic/Latino & $132(24.9 \%)$ & $66(22.3 \%)$ & $66(28.2 \%)$ & \\
\hline Black & $62(11.7 \%)$ & $33(11.1 \%)$ & $29(12.4 \%)$ & \\
\hline Asian & $79(14.9 \%)$ & $53(17.9 \%)$ & $26(11.1 \%)$ & \\
\hline Other & $102(19.2 \%)$ & $62(20.9 \%)$ & $40(17.1 \%)$ & \\
\hline $\begin{array}{l}\text { Percent of patients living in a zip code with at least } 25 \% \text { of residents } \\
\text { living below poverty line, +SD (missing } 40 \text { ) }\end{array}$ & $20.7 \%(13.7)$ & $21.1 \%(13.9)$ & $20.2 \%(13.6)$ & 0.49 \\
\hline Current cigarette smoking or vaping-no. (\%) & $18(3.4 \%)$ & $8(2.7 \%)$ & $10(4.3 \%)$ & 0.45 \\
\hline \multicolumn{5}{|l|}{ Severity of illness at presentation-no. (\%) } \\
\hline Emergency room only & $73(13.8 \%)$ & $40(13.5 \%)$ & $33(14.1 \%)$ & $<0.001$ \\
\hline Hospitalized without mechanical ventilation & $376(70.9 \%)$ & $229(77.4 \%)$ & $147(62.8 \%)$ & \\
\hline Hospitalized with mechanical ventilation & $81(15.3 \%)$ & $27(9.1 \%)$ & $54(23.1 \%)$ & \\
\hline Pre-COVID cardiopulmonary condition-no. (\%) & $320(60.4 \%)$ & $171(57.8 \%)$ & $149(63.7 \%)$ & \\
\hline Asthma & $55(10.4 \%)$ & $24(8.1 \%)$ & $31(13.2 \%)$ & 0.08 \\
\hline COPD or ILD & $19(3.6 \%)$ & $10(3.4 \%)$ & $9(3.8 \%)$ & 0.96 \\
\hline Heart failure & $23(4.3 \%)$ & $15(5.1 \%)$ & $8(3.4 \%)$ & 0.48 \\
\hline Coronary artery disease & $52(9.8 \%)$ & $33(11.1 \%)$ & $19(8.1 \%)$ & 0.31 \\
\hline Hypertension & $266(50.2 \%)$ & $151(51.0 \%)$ & $115(49.1 \%)$ & 0.73 \\
\hline Pre-COVID non-cardiopulmonary condition-no. (\%) & $90(17 \%)$ & $60(20.3 \%)$ & $30(12.8 \%)$ & \\
\hline Active cancer & $15(2.8 \%)$ & $8(2.7 \%)$ & $7(3.0 \%)$ & $>0.99$ \\
\hline Renal disease (CKD or ESRD) & $44(8.3 \%)$ & $33(11.1 \%)$ & $11(4.7 \%)$ & 0.012 \\
\hline Diabetes & $146(27.5 \%)$ & $87(29.4 \%)$ & $59(25.2 \%)$ & 0.33 \\
\hline HIV & $10(1.9 \%)$ & $3(1.0 \%)$ & $7(3.0 \%)$ & 0.12 \\
\hline Obesity (BMI >30) (missing 28) & $158(31.5 \%)$ & $76(27.0 \%)$ & $82(37.1 \%)$ & 0.021 \\
\hline Pregnancy & $15(2.8 \%)$ & $11(3.7 \%)$ & $4(1.7 \%)$ & 0.26 \\
\hline Organ transplant & $20(3.8 \%)$ & $15(5.1 \%)$ & $5(2.1 \%)$ & 0.13 \\
\hline Liver disease (cirrhosis or hepatitis) & $13(2.5 \%)$ & $10(3.4 \%)$ & $3(1.3 \%)$ & 0.21 \\
\hline Stroke or cerebrovascular disease & $28(5.3 \%)$ & $23(7.8 \%)$ & $5(2.1 \%)$ & 0.007 \\
\hline
\end{tabular}

Abbreviations: PASCpost-acute sequelae of COVID-19, BMI body mass index, CKD chronic kidney disease, COPD chronic obstructive pulmonary disease, ESRDend-stage renal disease, ILD interstitial lung disease, SD standard deviation ${ }^{\#} p<0.05$ in bold 

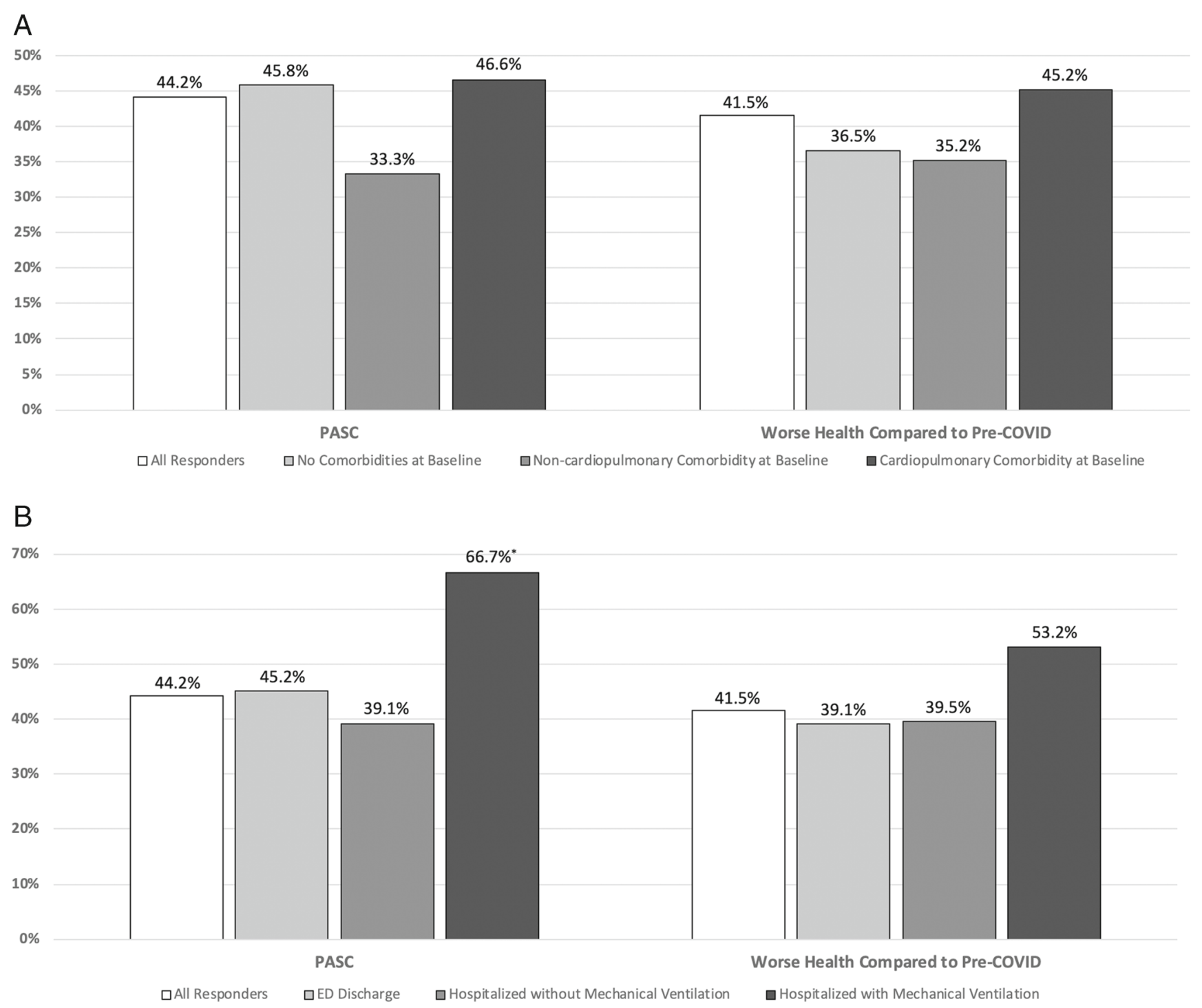

${ }^{*} p<0.05$ vs ED Discharge

Figure 1 Reported PASC symptoms and decline in health status from 1 year prior according to (A)pre-COVID comorbidity and (B) severity of acute COVID-19 illness

the most bothersome symptoms stratified by baseline comorbidity (no comorbidity vs non-cardiovascular vs cardiovascular) and severity of acute COVID-19 illness.

The results of the adjusted Poisson regression analysis revealed that women had a 1.26-fold higher risk of reporting persistent symptoms than men (Table 3). Those requiring mechanical ventilation during the index infection were also significantly more likely to experience PASC (aRR 1.48 [95\% CI 1.02-2.14]) as were people living with HIV (aRR 1.75 [95\% CI 1.14-2.69]). Additionally, patients with a baseline diagnosis of asthma had a trend toward higher PASC risk (aRR 1.27 [95\% CI 0.98-1.66]). Patients with a prior history of stroke (aRR 0.36 [95\% CI 0.15-0.86]) were less likely to experience PASC, and Asian patients (aRR 0.70 [95\% CI $0.49-1.02, p=0.06]$ ) and pregnant women (aRR 0.33 [95\% CI 1.14-2.69], $p=0.09$ ) had trends for lower PASC risk.
Notably, many risk factors for severe COVID-19 including age (both continuous and categorical) and several comorbid conditions were not independently associated with persistent symptoms at 1 year.

\section{Effort Tolerance}

Approximately one-third of the sample reported limitations in lifting/carrying groceries (36.2\%), climbing one flight of stairs (35.5\%), and bending/kneeling/stooping (38.1\%). Nearly one in four $(22.1 \%)$ reported limitations with walking one block. While limitations in effort tolerance were most significant among those with cardiopulmonary comorbid conditions, limitations in lifting/carrying groceries, climbing one flight of stairs, and bending/kneeling/stooping exceeded $20 \%$ among those without comorbid conditions (Figure 2A). While limitations in effort tolerance were also worse among those who 
Table 2 Most Bothersome Persistent Symptoms, Stratified by Pre-COVID Comorbid Conditions and Severity of Acute COVID-19 Infection

\begin{tabular}{|c|c|c|c|c|c|c|c|}
\hline & \multirow{2}{*}{$\frac{\text { All }}{(n=530)}$} & \multicolumn{3}{|c|}{ Pre-COVID comorbid conditions } & \multicolumn{3}{|c|}{ Severity of acute COVID-19 illness } \\
\hline & & $\begin{array}{l}\text { Cardio- } \\
\text { pulmonary } \\
(n=320)\end{array}$ & $\begin{array}{l}\text { Non- } \\
\text { cardiopulmonary } \\
(n=90)\end{array}$ & $\begin{array}{l}\text { None } \\
(n=120)\end{array}$ & $\begin{array}{l}\text { Mechanical } \\
\text { ventilation } \\
(n=81)\end{array}$ & $\begin{array}{l}\text { No mechanical } \\
\text { ventilation } \\
(n=376)\end{array}$ & $\begin{array}{l}\text { Discharged } \\
\text { from ED } \\
(n=73)\end{array}$ \\
\hline Brain fog & $20(3.8 \%)$ & $11(3.4 \%)$ & $1(1.1 \%)$ & $8(6.7 \%)$ & $3(3.7 \%)$ & $13(3.5 \%)$ & $4(5.5 \%)$ \\
\hline Cough & $10(1.9 \%)$ & $6(1.9 \%)$ & $0(0.0 \%)$ & $4(3.3 \%)$ & $1(1.2 \%)$ & $7(1.9 \%)$ & $2(2.7 \%)$ \\
\hline $\begin{array}{l}\text { Shortness } \\
\text { of breath }\end{array}$ & $55(10.4 \%)$ & $34(10.6 \%)$ & $9(10.0 \%)$ & $12(10.0 \%)$ & $10(12.3 \%)$ & $36(9.6 \%)$ & $9(12.3 \%)$ \\
\hline Neuropathy & $27(5.1 \%)$ & $17(5.3 \%)$ & $7(7.8 \%)$ & $3(2.5 \%)$ & $17(21.0 \%)$ & $9(2.4 \%)$ & $1(1.4 \%)$ \\
\hline Headache & $6(1.1 \%)$ & $4(1.2 \%)$ & $1(1.1 \%)$ & $1(0.8 \%)$ & $2(2.5 \%)$ & $4(1.1 \%)$ & $0(0.0 \%)$ \\
\hline Muscle aches & $16(3.0 \%)$ & $9(2.8 \%)$ & $3(3.3 \%)$ & $4(3.3 \%)$ & $2(2.5 \%)$ & $12(3.2 \%)$ & $2(2.7 \%)$ \\
\hline Diarrhea & $2(0.4 \%)$ & $2(0.6 \%)$ & $0(0.0 \%)$ & $0(0.0 \%)$ & $0(0.0 \%)$ & $1(0.3 \%)$ & $1(1.4 \%)$ \\
\hline Chest pain & $7(1.3 \%)$ & $5(1.6 \%)$ & $0(0.0 \%)$ & $2(1.7 \%)$ & $2(2.5 \%)$ & $3(0.8 \%)$ & $2(2.7 \%)$ \\
\hline $\begin{array}{l}\text { Abdominal } \\
\text { pain }\end{array}$ & $2(0.4 \%)$ & $2(0.6 \%)$ & $0(0.0 \%)$ & $0(0.0 \%)$ & $1(1.2 \%)$ & $1(0.3 \%)$ & $0(0.0 \%)$ \\
\hline $\begin{array}{l}\text { Loss of } \\
\text { smell/taste }\end{array}$ & $8(1.5 \%)$ & $5(1.6 \%)$ & $0(0.0 \%)$ & $3(2.5 \%)$ & $0(0.0 \%)$ & $6(1.6 \%)$ & $2(2.7 \%)$ \\
\hline Fatigue & $29(5.5 \%)$ & $19(5.9 \%)$ & $3(3.3 \%)$ & $7(5.8 \%)$ & $3(3.7 \%)$ & $23(6.1 \%)$ & $3(4.1 \%)$ \\
\hline Other & $47(8.9 \%)$ & $32(10.0 \%)$ & $6(6.7 \%)$ & $9(7.5 \%)$ & $13(16.0 \%)$ & $29(7.7 \%)$ & $5(6.8 \%)$ \\
\hline
\end{tabular}

Table 3 Adjusted Relative Risk Ratios and 95\% Confidence Intervals Testing Independent Associations Between Patient Characteristics at COVID-19 Presentation and Persistent Symptoms

\begin{tabular}{|c|c|c|}
\hline & $\begin{array}{l}\text { Adjusted relative risk } \\
\text { ratio* }[95 \% \mathrm{CI}]\end{array}$ & $p$-value ${ }^{\#}$ \\
\hline Age & $1.00[0.99-1.01]$ & 0.92 \\
\hline Female & $1.26[1.01-1.56]$ & 0.036 \\
\hline \multicolumn{3}{|l|}{ Race/ethnicity } \\
\hline White & Ref & - \\
\hline Hispanic/Latino & $1.04[0.80-1.36]$ & 0.77 \\
\hline Black & $1.01[0.72-1.42]$ & 0.94 \\
\hline Asian & $0.70[0.49-1.02]$ & 0.061 \\
\hline Other & $0.72[0.52-1.01]$ & 0.055 \\
\hline $\begin{array}{l}\text { Percent neighborhood } \\
\text { poverty rate }\end{array}$ & $1.00[0.99-1.01]$ & 0.50 \\
\hline \multicolumn{3}{|c|}{ Severity of acute COVID-19 illness } \\
\hline Emergency room only & Ref & - \\
\hline $\begin{array}{l}\text { Hospitalized, no mechanical } \\
\text { ventilation }\end{array}$ & $0.91[0.65-1.27]$ & 0.58 \\
\hline $\begin{array}{l}\text { Hospitalized, mechanical } \\
\text { ventilation }\end{array}$ & $1.48[1.02-2.14]$ & 0.038 \\
\hline \multicolumn{3}{|l|}{ Pre-COVID comorbidities } \\
\hline Asthma & $1.27[0.98-1.66]$ & 0.074 \\
\hline COPD or ILD & $0.82[0.47-1.44]$ & 0.49 \\
\hline Heart failure & $0.94[0.52-1.68]$ & 0.82 \\
\hline $\begin{array}{l}\text { Coronary artery disease } \\
\text { (CAD) }\end{array}$ & $0.85[0.57-1.25]$ & 0.40 \\
\hline Hypertension & $1.09[0.86-1.38]$ & 0.46 \\
\hline $\begin{array}{l}\text { Current tobacco use } \\
\text { (smoking or vaping) }\end{array}$ & $1.17[0.70-1.96]$ & 0.55 \\
\hline Active cancer & $1.13[0.58-2.18]$ & 0.72 \\
\hline $\begin{array}{l}\text { Renal disease (CKD or } \\
\text { ESRD) }\end{array}$ & $0.80[0.46-1.40]$ & 0.44 \\
\hline $\begin{array}{l}\text { ESRD) } \\
\text { Diabetes }\end{array}$ & $0.93[0.73-1.18]$ & 0.56 \\
\hline HIV & $1.75[1.14-2.69]$ & 0.010 \\
\hline $\begin{array}{l}\text { Obesity }(\mathrm{BMI}>30) \text { (missing } \\
\text { for } 76 \text { ) }\end{array}$ & $1.09[0.87-1.36]$ & 0.47 \\
\hline Pregnancy & $0.33[0.09-1.18]$ & 0.089 \\
\hline Any organ transplant & $0.61[0.25-1.47]$ & 0.27 \\
\hline $\begin{array}{l}\text { Liver disease (cirrhosis or } \\
\text { hepatitis) }\end{array}$ & $0.74[0.28-1.98]$ & 0.55 \\
\hline $\begin{array}{l}\text { Stroke or cerebrovascular } \\
\text { disease }\end{array}$ & $0.36[0.15-0.86]$ & 0.022 \\
\hline
\end{tabular}

Abbreviations: BMI body mass index, CKD chronic kidney disease, COPD chronic obstructive pulmonary disease, ESRDend-stage renal disease, ILD interstitial lung disease, SD standard deviation

*Adjusted for age, sex, race, poverty, co-morbidities, severity of illness ${ }^{\#} p<0.10$ in bold required hospitalization and mechanical ventilation, limitations in lifting/carrying groceries, climbing one flight of stairs, and bending/kneel exceeded $25 \%$ even among those who only required an ED visit without overnight hospitalization (Figure 2B and Supplemental Table 2).

\section{DISCUSSION}

Nearly one year after their acute infection, a substantial number of the diverse patients in this study reported worse health status compared to the prior year; persistent symptoms, especially shortness of breath and fatigue; and significant limitations carrying out simple tasks such as carrying groceries and walking one block. To our knowledge, this is among the first data examining these important patient-reported parameters approximately 1 year after COVID-19 infection in the USA, providing early insights on the long-term impact on several aspects of health.

Importantly, we found that declines in health status, persistent symptoms, and limitations in effort tolerance occurred in substantial proportions of patients regardless of baseline comorbidity burden and/or severity of the acute COVID-19 illness. Our findings suggest that while relatively healthy people may be spared from severe COVID-19, they may not be protected from the long-term effects of COVID-19. Unlike other complications related to acute COVID- $19,{ }^{9}$ we found that age was not a risk factor for PASC. Findings such as these merit dissemination so that even healthy adults can make informed decisions about the risks and benefits of vaccination. Although there are limited data on whether vaccinations lower risks for PASC, should patients contract breakthrough infections, preventing COVID-19 in the first place through continued mask wearing and vaccination may be the best line of defense against PASC, a condition that appears to be extraordinarily common and debilitating. 

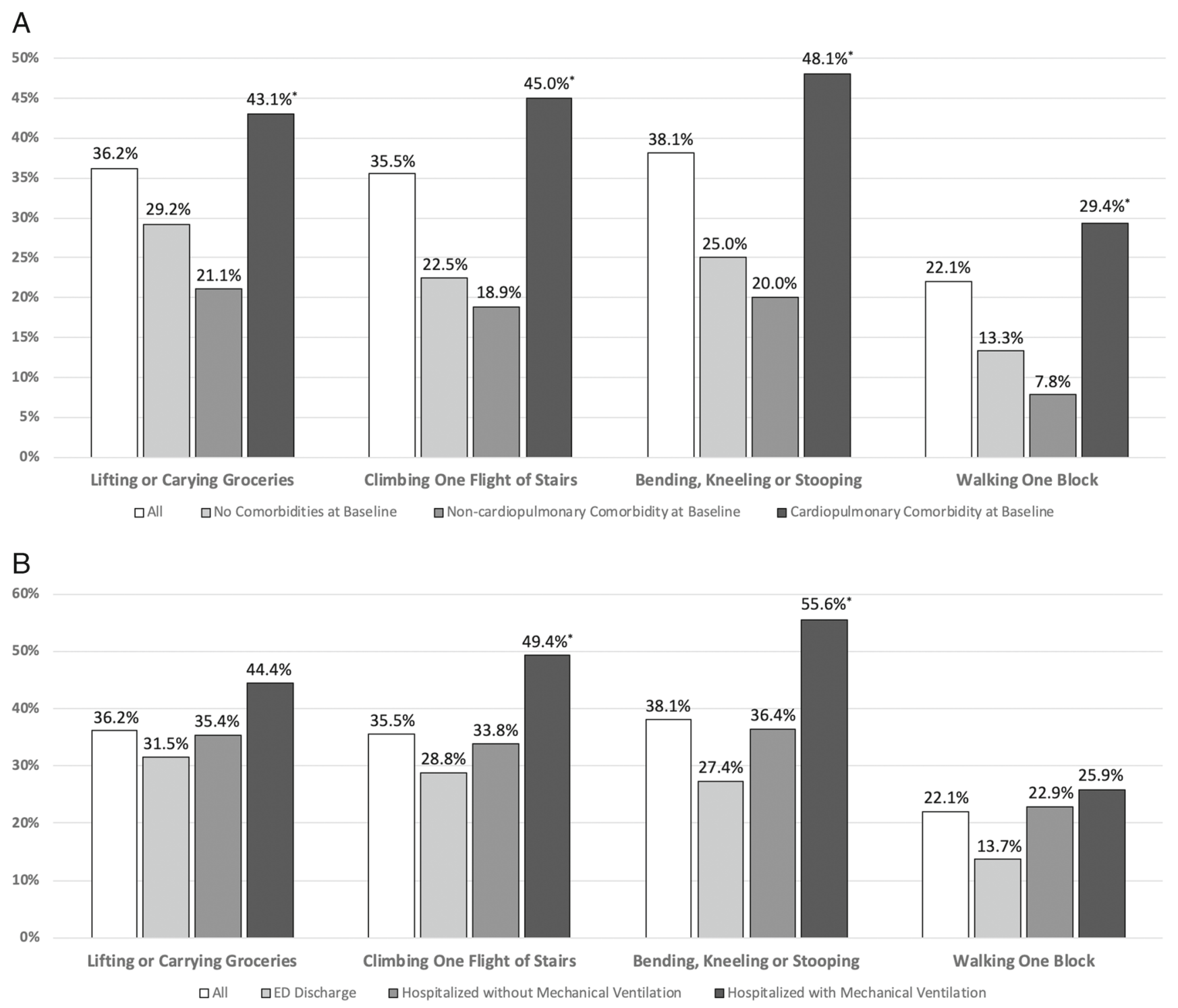

${ }^{*} p<0.05$ vs No Comorbidities or ED Discharge, respectively

Figure 2 Limitations in effort tolerance, according to (A) baseline comorbidity strata and (B) severity of acute COVID-19 illness strata

Our findings suggest that women may be more vulnerable to PASC, a finding that builds upon our previous report indicating a trend toward higher return to the Emergency Department among women in the first 30 days after hospital discharge. ${ }^{10}$ Interestingly, in the acute phase of illness, the converse is true - both infection rates and mortality are higher in men compared to women. Mechanistic underpinnings of this observation are not known but suggest that the risk factors for severe COVID-19 differ from those for PASC. It is possible that the greater immunoregulation leading to improved initial outcome in women leads to a longer-term chronic process. More specifically, studies indicate that women elicit much stronger immune responses across a myriad of exposures, including viruses. ${ }^{11}$ After HIV infection, women have significantly lower serum viral load. ${ }^{12}$ Additionally, women develop significantly higher serum antibody levels after vaccination against most viral targets, including COVID-19. ${ }^{13}, 14$ This said, women also account for approximately $80 \%$ of all long-term autoimmune diseases. ${ }^{15}$ More specific to acute COVID-19, while testosterone and other Y-linked pathways (such as TMPRSS2, Th17) have been shown to increase SARS-CoV2 entry and disease severity in men, both $\mathrm{X}$-linked promoter regions and estrogen in females suppress these same pathways, thereby limiting damage during initial infection. ${ }^{16}$ Therefore, we speculate that while the initial virostatic burst in women may suppress processes such as viral entry and/or replication, it may in turn lead to longterm but low-level immune activation and subsequent PASC. This said, it is also possible that this finding is due to survivor bias from the acute phase. Our findings here support the urgent need to further understand the pathophysiology of PASC, and subsequently develop patient-specific treatment strategies for this debilitating condition. 
More than a year after the COVID-19 pandemic enveloped the globe, its true impact is only emerging. The full costs of COVID-19 are yet to be realized, as our data suggest that the effects of COVID-19 extend well beyond the acute phase and are likely to have a major impact on several sectors of the workforce as well as the well-being of many families. Our data underscore that much work remains to be done and they serve as a caution to those who continue to defer vaccination against this terrible disease.

Strengths of this study include its relatively large size and the diversity of the sample. We obtained data by speaking directly to patients for outcome detection rather than relying on medical records that may lack sensitivity, since they require medical follow-up. We also had data to characterize the index infection that were abstracted from medical records, enhancing reliability. Limitations include the setting of only two hospitals in one city, possibly impacting generalizability and lack of objective functional measures such as pulmonary function tests and echocardiography. Future work incorporating objective physiologic data are necessary to better understand mechanisms underlying PASC. Since nearly $50 \%$ were lost to follow-up, there is concern that these data may underestimate PASC, especially in specific groups noted to be underrepresented in our data such as Black patients, patients with active cancer, and those actively using tobacco. For example, those with active cancer may have died before the survey was administered, which could have biased results. Last, we observed a trend suggesting that milder cases may have lower risk for PASC, suggesting that studies including mild and asymptomatic cases are needed to estimate risks for the entire spectrum of COVID-19 survivors.

In conclusion, PASC may be extraordinarily common 1 year after moderate to severe COVID-19, and these symptoms are sufficiently severe to substantially impact the exercise tolerance of patients in their daily lives. Further, unlike acute COVID-19, PASC symptoms are broadly distributed, are not limited to one specific patient group, and appear to be unrelated to age. These data have implications for vaccine hesitant individuals, policy makers, and physicians managing the emerging longer-term yet unknown impact of the COVID-19 pandemic.

Supplementary Information The online version contains supplementary material available at https://doi.org/10.1007/s11606-021O7379-Z.

Acknowledgements: The authors would like to formally acknowledge our chart abstraction team, which included Weill Cornell Medicine medical students and New York-Presbyterian/Weill Cornell Medical Center house staff. This study received support from New York-Presbyterian Hospital (NYPH) and Weill Cornell Medical College (WCMC), including the Clinical and Translational Science Center (CTSC) (UL1 TROO0457) and Joint Clinical Trials Office (JCTO).

Corresponding Author: Justin R. Kingery, MD,PhD; Division of General Internal Medicine, Department of Medicine, Weill Medical College of Cornell University, 402 E. 67th Street, 2nd Floor, New York, NY 10065, USA (e-mail: jrk9006@med.cornell.edu).
Funding Dr. Kingery is supported by a National Institute of Health/ National Heart Lung Blood Institute grant (K23-HL-152926). Dr. Safford consults for Amgen. Dr. Goyal is supported by American Heart Association grant 20CDA35310455, National Institute on Aging grant K76AG064428, and Loan Repayment Program award L30AG060521; Dr. Goyal receives personal fees for medicolegal consulting related to heart failure, and has received honoraria from Akcea Inc. and Bionest Inc. Dr McNairy is supported by a National Heart Lung Blood Institute grant (R01-143788).

\section{Declarations:}

Conflict of Interest: Dr. Kingery is supported by a National Institute of Health/National Heart Lung Blood Institute grant (K23-HL-152926). Dr. Safford consults for Amgen. Dr. Goyal is supported by American Heart Association grant 20CDA35310455, National Institute on Aging grant K76AG064428, and Loan Repayment Program award L30AG060521; Dr. Goyal receives personal fees for medicolegal consulting related to heart failure, and has received honoraria from Akcea Inc. and Bionest Inc. Dr McNairy is supported by a National Heart Lung Blood Institute grant (RO1-143788).

\section{REFERENCES}

1. Group TRC. Dexamethasone in Hospitalized Patients with Covid-19. N Engl J Med. 2021;

2. Nasserie T, Hittle M, Goodman SN. Assessment of the Frequency and Variety of Persistent Symptoms among Patients with COVID-19: A Systematic Review. JAMA Netw. Open. 2021;

3. Nehme M, Braillard O, Chappuis F, Courvoisier DS, Guessous I. Prevalence of Symptoms More Than Seven Months After Diagnosis of Symptomatic COVID-19 in an Outpatient Setting. Ann Intern Med. 2021;

4. Huang C, Huang L, Wang Y, Li X, Ren L, Gu X, Kang L, Guo L, Liu M, Zhou X, Luo J, Huang Z, Tu S, Zhao Y, Chen L, Xu D, Li Y, Li C, Peng L, Li Y, Xie W, Cui D, Shang L, Fan G, Xu J, Wang G, Wang Y, Zhong J, Wang C, Wang J, Zhang D, Cao B. 6-month consequences of COVID-19 in patients discharged from hospital: a cohort study. Lancet. 2021;

5. Goyal P, Choi JJ, Pinheiro LC, Schenck EJ, Chen R, Jabri A, Satlin MJ, Campion TR, Nahid M, Ringel JB, Hoffman KL, Alshak MN, Li HA, Wehmeyer GT, Rajan M, Reshetnyak E, Hupert N, Horn EM, Martinez FJ, Gulick RM, Safford MM. Clinical Characteristics of Covid19 in New York City. N Engl J Med. 2020;

6. Taboada M, Moreno E, Cariñena A, Rey T, Pita-Romero R, Leal S, Sanduende Y, Rodriguez A, Nieto C, Vilas E, Ochoa M, Cid M, SeoanePillado T. Quality of life, functional status, and persistent symptoms after intensive care of COVID-19 patients. Br. J. Anaesth. 2021;

7. National Center for Immunization and Respiratory Diseases D of VD. Centers for Disease Control: COVID-19 [Internet]. 2021;Available from: https://www.cdc.gov/coronavirus/2019-ncov/need-extra-precautions / people-with-medical-conditions.html

8. Knol MJ, Le Cessie S, Algra A, Vandenbroucke JP, Groenwold RHH. Overestimation of risk ratios by odds ratios in trials and cohort studies: Alternatives to logistic regression. CMAJ. 2012;

9. O'Driscoll M, Ribeiro Dos Santos G, Wang L, Cummings DAT, Azman AS, Paireau J, Fontanet A, Cauchemez S, Salje H. Age-specific mortality and immunity patterns of SARS-CoV-2. Nature. 2021;

10. Kingery JR, BF Martin P, Baer BR, Pinheiro LC, Rajan M, Clermont A, Pan S, Nguyen K, Fahoum K, Wehmeyer GT, Alshak MN, Li HA, Choi JJ, Shapiro MF, McNairy ML, Safford MM, Goyal P. Thirty-Day Post-Discharge Outcomes Following COVID-19 Infection. J Gen Intern Med. 2021;

11. Klein SL, Flanagan KL. Sex differences in immune responses. Nat. Rev. Immunol. 2016;

12. Sterling TR, Vlahov D, Astemborski J, Hoover DR, Margolick JB, Quinn TC. Initial Plasma HIV-1 RNA Levels and Progression to AIDS in Women and Men. N Engl J Med. 2001;

13. Klein SL, Jedlicka A, Pekosz A. The Xs and Y of immune responses to viral vaccines. Lancet Infect. Dis. 2010;

14. Amodio E, Capra G, Casuccio A, De Grazia S, Genovese D, Pizzo S, Calamusa G, Ferraro D, Giammanco GM, Vitale F, Bonura F. Antibodies responses to sars-cov-2 in a large cohort of vaccinated subjects and seropositive patients. Vaccines. 2021; 
15. Invernizzi P, Pasini S, Selmi C, Gershwin ME, Podda M. Female predominance and $\mathrm{X}$ chromosome defects in autoimmune diseases. J. Autoimmun. 2009;

16. Kumar A, Narayan R, Kulandhasamy M, Prasoon P, Kumari C, Kumar S, Pareek V, Sesham K, Shekhawat PS, Kant K KS. COVID-19 pandemic: insights into molecular mechanisms leading to sex-based differences in patient outcomes. Expert Rev Mol Med. 2021;23.
Publisher's Note: Springer Nature remains neutral with regard to jurisdictional claims in published maps and institutional affiliations. 